\title{
Therapeutic effect of ascorbic acid on dapsone-induced methemoglobinemia in rats
}

\author{
Changwoo Kang ${ }^{1,2}$, Dong Hoon Kim ${ }^{1,2}$, Taeyun Kim ${ }^{1}$, Soo Hoon Lee ${ }^{1,2}$, \\ Jin Hee Jeong ${ }^{1}$, Sang Bong Lee ${ }^{1}$, Jin Hyun Kim ${ }^{2,3}$, Myeong Hee Jung ${ }^{3}$, \\ Kyung-woo Lee ${ }^{4}$, In Sung Park ${ }^{2,5}$
}

\begin{abstract}
'Department of Emergency Medicine, Gyeongsang National University Hospital, Gyeongsang National University School of Medicine, Jinju, Korea

${ }^{2}$ Institute of Health Sciences, Gyeongsang National University, Jinju, Korea

${ }^{3}$ Biomedical Research Institute, Gyeongsang National University Hospital, Jinju, Korea

${ }^{4}$ Department of Emergency Medicine, Catholic University of Daegu, Daegu, Korea

${ }^{5}$ Department of Neurosurgery, Gyeongsang National University Hospital, Gyeongsang National University

School of Medicine, Jinju, Korea
\end{abstract}

Objective Dapsone (diaminodiphenyl sulfone, DDS) is currently used to treat leprosy, malaria, dermatitis herpetiformis, and other diseases. It is also used to treat pneumocystis pneumonia and Toxoplasma gondii infection in HIV-positive patients. The most common adverse effect of DDS is methemoglobinemia from oxidative stress. Ascorbic acid is an antioxidant and reducing agent that scavenges the free radicals produced by oxidative stress. The present study aimed to investigate the effect of ascorbic acid in the treatment of DDS induced methemoglobinemia.

Methods Male Sprague-Dawley rats were divided into three groups: an ascorbic acid group, a methylene blue (MB) group, and a control group. After DDS (40 mg/kg) treatment via oral gavage, ascorbic acid $(15 \mathrm{mg} / \mathrm{kg}), \mathrm{MB}(1 \mathrm{mg} / \mathrm{kg})$, or normal saline were administered via tail vein injection. Depending on the duration of the DDS treatment, blood methemoglobin levels, as well as the nitric oxide levels and catalase activity, were measured at 60,120, or 180 minutes after DDS administration.

Results Methemoglobin concentrations in the ascorbic acid and MB groups were significantly lower compared to those in the control group across multiple time points. The plasma nitric oxide levels and catalase activity were not different among the groups or time points.

Conclusion Intravenous ascorbic acid administration is effective in treating DDS-induced methemoglobinemia in a murine model.

Keywords Methemoglobin; Ascorbic acid; Methylene blue; Anti-oxidation

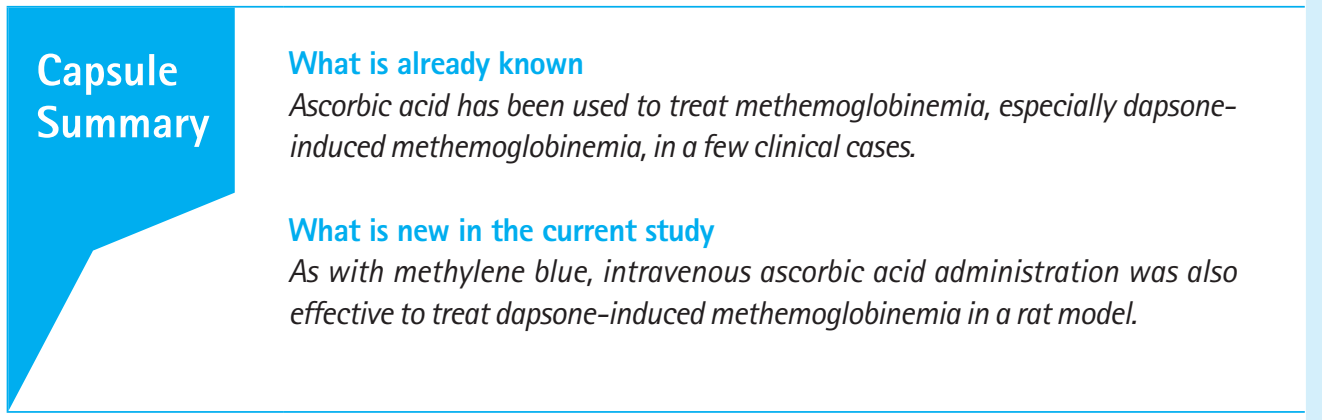

elSSN: 2383-4625

Received: 13 July 2017

Revised: 17 October 2017

Accepted: 29 October 2017

Correspondence to: In Sung Park Department of Neurosurgery, Gyeongsang National University Hospital, Gyeongsang National University School of Medicine, 79 Gangnam-ro, Jinju 52727, Korea E-mail: gnuhpis@gnu.ac.kr

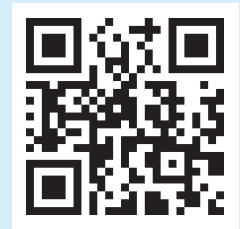

How to cite this article:

Kang C, Kim DH, Kim T, Lee SH, Jeong JH, Lee SB, Kim JH, Jung MH, Lee KW, Park IS. Therapeutic effect of ascorbic acid on dapsone-induced methemoglobinemia in rats. Clin Exp Emerg Med 2018;5(3):192198.

This is an Open Access article distributed under the terms of the Creative Commons Attribution Non-Commercial License (http:// creativecommons.org/licenses/by-nc/4.0/). 


\section{INTRODUCTION}

Dapsone (diaminodiphenyl sulfone, DDS) is a sulfone antibiotic that decreases folate synthesis by inhibiting dihydropteroate synthetase. ${ }^{1,2}$ It is commonly used to treat leprosy, malaria, dermatitis herpetiformis, and other diseases, and has also been administered as a prophylactic treatment of pneumocystis pneumonia and Toxoplasma gondii infection in HIV-positive patients., ${ }^{3,4}$

DDS is insoluble in water and is readily absorbed in the gastrointestinal tract. The peak plasma concentration after oral administration of DDS is reached at 2 to 6 hours after a dose of $100 \mathrm{mg} /$ $\mathrm{kg}$, and the half-life of the drug is 20 to 30 hours. ${ }^{1,5-7}$ DDS is metabolized via either $\mathrm{N}$-hydroxylation or acetylation through portal circulation in the liver (Fig. 1). It has been suggested that the most common adverse effects of DDS-methemoglobinemia and hemolysis-are induced by DDS-hydroxylamine, an N-hydroxylated metabolite of DDS that produces a number of reactive oxygen species and methemoglobin as a result of a cyclic oxidation-reduction reaction between DDS-hydroxylamine and oxyhemoglobin in red blood cells. s. $^{8-10}$

Methemoglobin contains one or more ferric state heme ions oxidized from ferrous ions; accordingly, it is incapable of binding

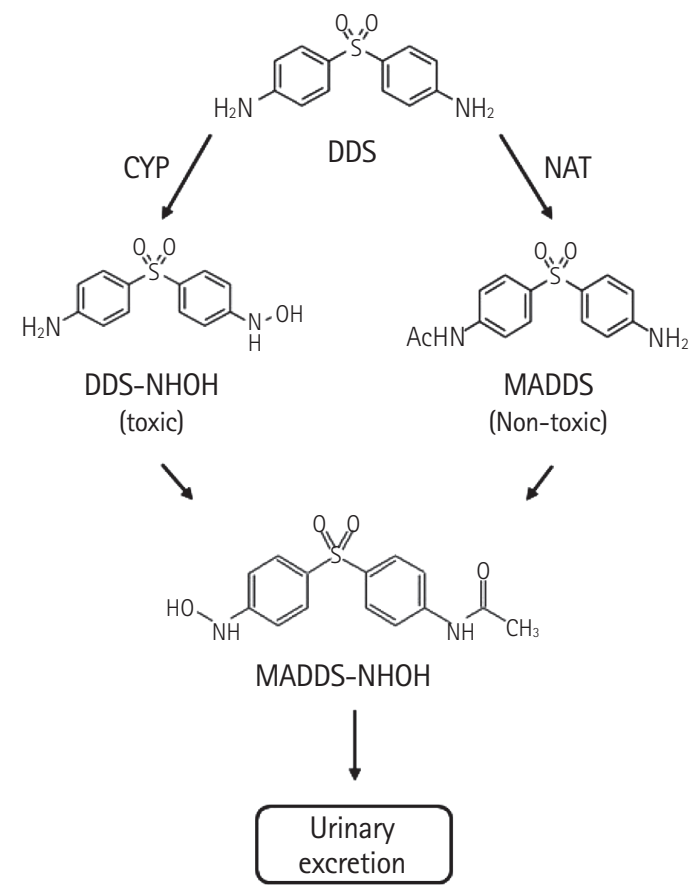

Fig. 1. Metabolic pathways of dapsone (DDS) after absorption from the gastrointestinal tract. DDS is transported to the liver, where it is metabolized via either N-hydroxylation or acetylation. CYP, cytochrome P450; NAT, N-acetyl transferase; DDS-NHOH, dapsone hydroxylamine; MADDS, monoacetyl dapsone; MADDS- $\mathrm{NHOH}$, monoacetyl dapsone hydroxylamine. to oxygen. ${ }^{11,12}$ Therefore, when the concentration of methemoglobin in the blood is elevated, tissue hypoperfusion, and/or hypoxia can occur. Severe methemoglobinemia resulting from a DDS overdose can cause cyanosis, dizziness, dyspnea, tachycardia, altered mental status, and eventually, death. ${ }^{13-16}$

The most widely accepted treatment for methemoglobinemia is intravenous injection of methylene blue. This compound is reduced to colorless leucomethylene blue by nicotinamide adenine dinucleotide phosphate (NADPH) methylene blue reductase, which then reduces methemoglobin to hemoglobin. ${ }^{10}$ However, methylene blue could induce hemolysis, and should not be administered to patients with known glucose-6-phosphate dehydrogenase (G6PD) deficiency and non-G6PD deficiency infants. ${ }^{17,18}$ As an alternative to methylene blue, ascorbic acid has been used to treat methemoglobinemia, although multiple doses are required, and its response is very slow. ${ }^{19-21}$

Methylene blue has been unavailable in most Korean emergency departments because of an import suspension over the past few years. Therefore, we have used ascorbic acid to treat methemoglobinemia. The present study was performed to investigate the effects of ascorbic acid in the treatment of DDS-induced methemoglobinemia by comparing its activity to that of methylene blue in an animal model.

\section{METHODS}

\section{Study design}

This study consisted of three sets of time dependent experiments $(60,120$, and 180 minutes) of DDS treatment. The Institutional Animal Care and Use Committee of our university approved all the protocols (GNU-150508-R0029). This experiment was conducted in accordance with Guide for the Care and Use of Laboratory Animals prepared by the National Academy of Sciences.

\section{Animal preparation}

Forty-five drug-naïve male Sprague-Dawley rats weighing 250 to $300 \mathrm{~g}$ were used. The animals were housed in a controlled environment for 3 to 7 days and were allowed free access to food and water. All animals were fasted for 8 hours before the experiment, but had free access to water. The rats were anesthetized by inhalation of 3\% isoflurane using an anesthetizing box for 60 to 90 seconds. The tail vein was cannulated using a 24-gauge catheter (BD Insyte Autoguard; Becton-Dickinson, Franklin Lake, NJ, USA) to administer the medication. DDS ( $40 \mathrm{mg} / \mathrm{kg}$; Santa Cruz Biotechnology, Santa Cruz, CA, USA) in dimethyl sulfoxide (1 mg/ $\mathrm{kg}$, Santa Cruz Biotechnology) was administered to the rats via oral gavage to induce poisoning. 


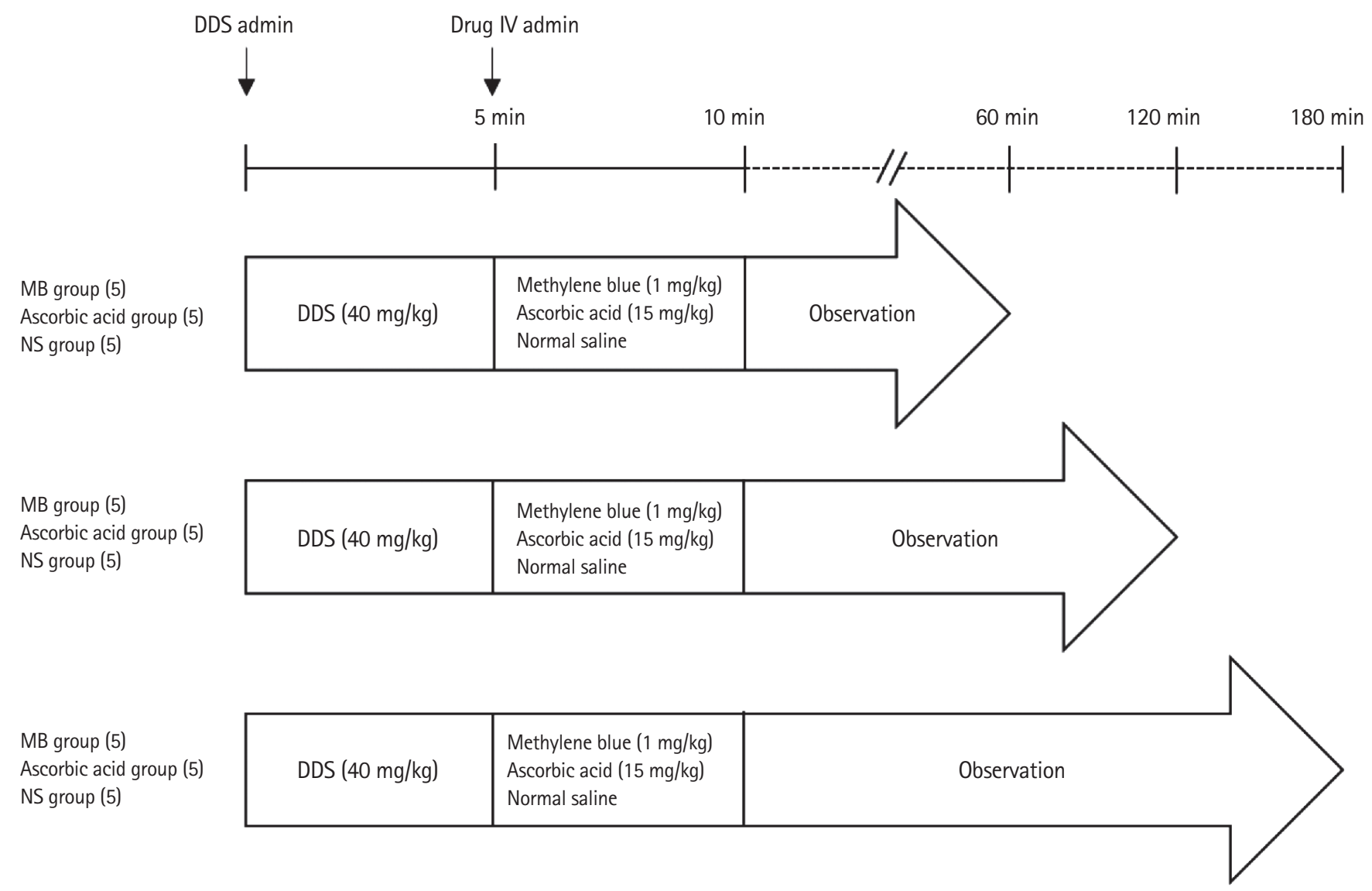

Fig. 2. Study protocol of the animal experiment. DDS, dapsone; IV, intravenous; MB, methylene blue; NS, normal saline.

\section{Study protocol}

The rats were divided into an ascorbic acid group, a methylene blue group, and a control group. Five minutes after DDS administration, ascorbic acid (15 mg/kg; Huons, Seongnam, Korea), methylene blue (1 mg/kg; Akorn, Lake Forest, IL, USA), or normal saline was administered via tail vein injection over a 5-minute period (depending on the group) using a syringe pump (IVAC P6000; Alaris Medical Systems, San Diego, CA, USA). Ascorbic acid and methylene blue were diluted in normal saline, and equivalent volumes were administered to the three groups. At 60, 120, and 180 minutes after DDS administration, five rats from each group were anesthetized using an intravenous injection of alfaxalone (10 mg/kg; Alfaxan, Jurox, Rutherford, NSW, Australia) (Fig. 2).

\section{Blood collection}

After anesthesia, a thoracotomy was performed, and blood samples were collected from the heart of the rats. The blood samples were divided into a heparinized syringe to measure the methemoglobin levels and into an EDTA tube for the nitric oxide (NO) and catalase (CAT) assays. Plasma separation by centrifugation at
3,000 rpm for 15 minutes at $4^{\circ} \mathrm{C}$ was performed, and the plasma was stored at $-80^{\circ} \mathrm{C}$ until the NO and CAT assays were conducted.

\section{Biochemical measurements \\ Blood methemoglobin level}

The methemoglobin concentration in the blood was measured using an ABL90 FLEX blood gas analyzer (Radiometer, Copenhagen, Denmark). Carbon monoxide oximetry was used to determine the percentage of methemoglobin in a blood sample.

Determination of oxidative stress parameters (NO and CAT assays)

$\mathrm{NO}$ is a reactive radical that rapidly oxidizes to nitrite and nitrate. NO levels were measured based on the concentrations of accumulated nitrate and nitrite using a modified Griess method with a commercial quantitative colorimetric assay kit (QuantiChrom Nitric Oxide Assay Kit, BioAssay Systems, Hayward, CA, USA) according to the manufacturer's instructions.

Briefly, $150 \mu \mathrm{L}$ of plasma was mixed with $8 \mu \mathrm{L}$ of $\mathrm{ZnSO}_{4}$ in 1.5 $\mathrm{mL}$ tubes. After the samples were vortexed, $8 \mu \mathrm{L}$ of $\mathrm{NaOH}$ was 
added, and the samples were vortexed again and centrifuged for 10 minutes at $14,000 \mathrm{rpm}$. Approximately $100 \mu \mathrm{L}$ of the clear supernatant was transferred to a clean tube. Then, $100 \mu \mathrm{L}$ of reagent $A, 4 \mu \mathrm{L}$ of reagent $B$, and $100 \mu \mathrm{L}$ of reagent $C$ were mixed, and $200 \mu \mathrm{L}$ of the working solution was added to each sample and incubated for 10 minutes at $60^{\circ} \mathrm{C}$. The reaction tubes were centrifuged to pellet any precipitates, and $250 \mu \mathrm{L}$ of each reaction was transferred to separate wells in a 96-well plate. The optical density of the samples was measured at $540 \mathrm{~nm}$.

CAT is an antioxidant enzyme that converts hydrogen peroxide $\left(\mathrm{H}_{2} \mathrm{O}_{2}\right)$ to water and oxygen. CAT activity was measured using the EnzyChrom Catalase Assay Kit (BioAssay Systems).

Ten microliters of each sample was transferred into the wells of a 96-well plate. One sample was prepared as a blank well and contained only $10 \mu \mathrm{L}$ of assay buffer. Next, $400 \mu \mathrm{L}$ of assay buffer was added to positive control tube and mixed well; $10 \mu \mathrm{L}$ of this reconstituted positive control was transferred into a separate well. Thereafter, $5 \mu \mathrm{L}$ of $3 \% \mathrm{H}_{2} \mathrm{O}_{2}$ and $914 \mu \mathrm{L}$ of $\mathrm{dH}_{2} \mathrm{O}$ (final concentration $4.8 \mathrm{mM}$ ) were mixed, and $90 \mu \mathrm{L}$ of this $50 \mu \mathrm{M}$ substrate was added to these wells to initiate the CAT reaction and incubated for 30 minutes at room temperature. Afterwards, $40 \mu \mathrm{L}$ of 4.8 $\mathrm{mM} \mathrm{H} \mathrm{O}_{2}$ was combined with $440 \mu \mathrm{L}$ of $\mathrm{dH}_{2} \mathrm{O}$ to yield $400 \mu \mathrm{M}$ $\mathrm{dH}_{2} \mathrm{O}_{2}$. For each well, $102 \mu \mathrm{L}$ of assay buffer, $1 \mu \mathrm{L}$ of dye reagent, and $1 \mu \mathrm{L}$ of horseradish peroxidase was added, followed by the addition of $100 \mu \mathrm{L}$ of detection reagent per well after a 30-minute incubation. After the samples were incubated for 10 minutes, their optical density values were measured at $570 \mathrm{~nm}$.

\section{Statistical analysis}

The sample size was calculated based on superiority of intravenous ascorbic acid and methylene blue administration over nor-

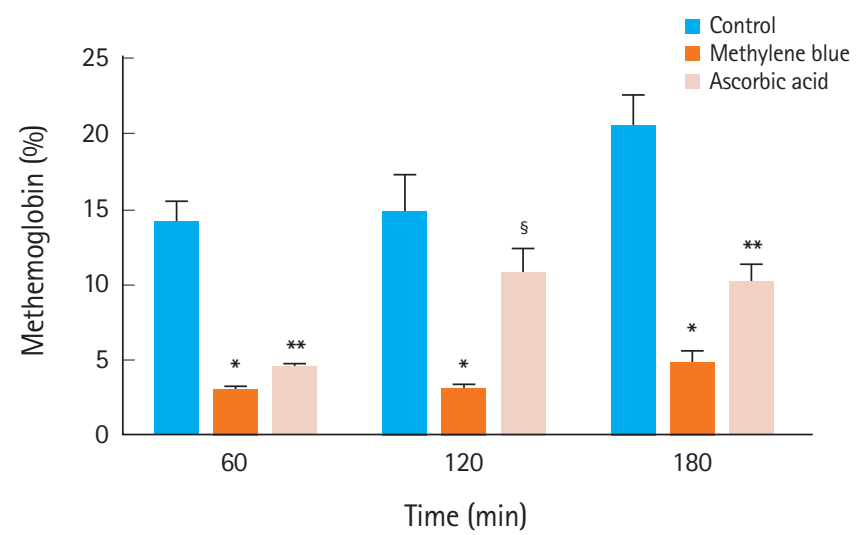

Fig. 3. Methemoglobin concentrations among the three groups at 60 , 120 , and 180 minutes after dapsone treatment. ${ }^{*} P<0.01$, methylene blue group vs. control group. ${ }^{* *} \mathrm{P}<0.01$, ascorbic acid group vs. control group. ${ }^{\S} \mathrm{P}=0.01$, methylene blue group vs. ascorbic acid group. mal saline in DDS-induced methemoglobinemia. On the basis of a previous study, we expected that ascorbic acid and methylene blue administration would be approximately $40 \%$ more effective compared to normal saline at lowering the levels of methemoglobin. ${ }^{22}$ A total sample size of 15 rats (five in each group) achieves a 90\% power to detect a difference of 5\% among the means.

Continuous data are expressed as the mean \pm standard deviation. The test for normality was performed using a Shapiro-Wilk test. The Kruskal-Wallis test with the Mann-Whitney U post hoc test was used to perform comparisons among the groups if the normality assumption was not satisfied. Statistical analysis was conducted using IBM SPSS ver. 21.0 (IBM Corp., Armonk, NY, USA), and P-values $<0.05$ were considered statistically significant.

\section{RESULTS}

\section{Methemoglobin concentration}

The methemoglobin concentrations in the three groups at 60, 120, and 180 minutes are shown in Fig. 3. At 60 minutes, significantly lower concentrations of methemoglobin were measured in the methylene blue $(3.0 \pm 0.7 \%)$ and ascorbic acid groups $(4.4 \pm 1.0 \%)$ compared with the concentrations in the control group $(14.1 \pm 3.2 \%$, $\mathrm{P}<0.01)$. At 120 minutes, the methemoglobin concentration was $14.8 \pm 5.5 \%$ in the control group, $3.1 \pm 0.8 \%$ in the methylene blue group, and $10.7 \pm 3.6 \%$ in the ascorbic acid group $(P=0.01)$. At 180 minutes, the methemoglobin concentrations of three groups were $20.4 \pm 4.7 \%$ (control group), $4.8 \pm 1.7 \%$ (methylene blue group), and $10.0 \pm 3.0 \%$ (ascorbic acid group) $(\mathrm{P}<0.01)$.

\section{NO levels and CAT activity}

The plasma NO levels of the three groups at 60, 120, and 180

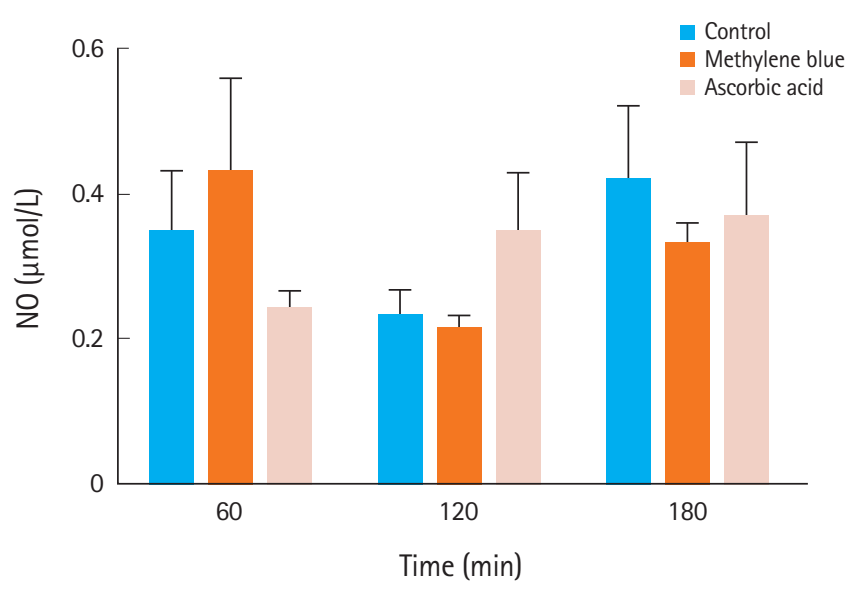

Fig. 4. Plasma nitric oxide (NO) levels among the three groups at 60,120 and 180 minutes after dapsone treatment. 


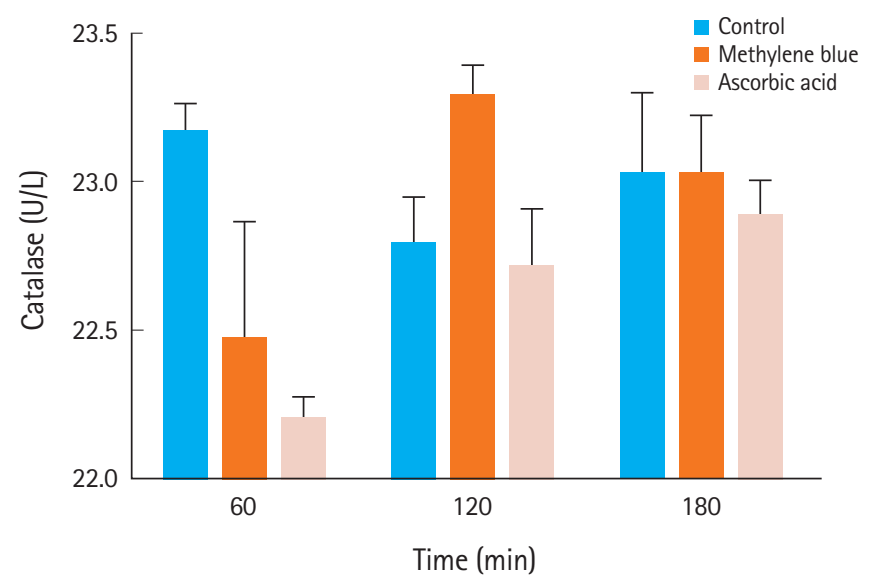

Fig. 5. Catalase activity among the three groups at 60,120 , and 180 minutes after dapsone treatment.

minutes are shown in Fig. 4. There were no statistically significant differences among the three groups. The CAT activity in the three groups at 60,120, and 180 minutes is shown in Fig. 5. There were also no statistically significant differences among the groups.

\section{DISCUSSION}

Based on our results, ascorbic acid is an effective treatment for methemoglobinemia induced by DDS poisoning. However, it is unclear whether ascorbic acid is more effective than methylene blue at treating DDS-induced methemoglobinemia.

Methemoglobin is formed by oxidizing normal ferrous heme ions to ferric state ions. Methemoglobin has a reduced ability for oxygen transport to tissues, which causes cellular hypoxia. Under normal conditions, methemoglobin levels are $<1 \%$. However, under conditions of oxidative stress, methemoglobin levels can increase. There are two types of methemoglobinemia: congenital and acquired. Acquired methemoglobinemia is a result of increased methemoglobin formation by exogenous oxidizing agents and is more common; cases of congenital methemoglobinemia are rare.

Normally, low methemoglobin levels are maintained by reducing mechanisms through enzymatic systems and cellular antioxidants. NADH-dependent cytochrome 65 methemoglobin reductase is the major enzyme responsible for the reduction of methemoglobin, and NADPH-dependent methemoglobin reductase is a minor pathway. ${ }^{10}$ Cellular antioxidants, such as ascorbic acid and glutathione, play a minor role in reducing methemoglobin.

If significant symptoms (e.g., cyanosis, dizziness, dyspnea, tachycardia, or altered mental status) indicative of methemoglobinemia are present, or methemoglobin levels are $>25 \%$, which is often the situation in intentional or accidental drug overdoses, specific therapies are indicated. The standard treatment for methemoglo- binemia is intravenous administration of methylene blue, which is currently considered the gold standard of treatment for methemoglobinemia. Methylene blue reduces toxic levels of methemoglobin to a non-toxic level within 10 to 60 minutes via an NADPHdependent pathway. ${ }^{23}$

However, methylene blue should not be administered to patients with known G6PD deficiency, because it reduces the NADPH generated by G6PD. As a result, this treatment is minimally effective, or could be potentially harmful, because it may exacerbate the degree of methemoglobinemia and induce acute hemolysis. In addition, methylene blue can cause serotonin toxicity. Several case reports of methylene blue-associated serotonin toxicity have been described in which methylene blue was intravenously administered to stain parathyroid glands during parathyroidectomy in patients receiving serotonergic psychotropic drugs. ${ }^{24}$ In one severe case, methylene blue-associated serotonin toxicity was fatal. ${ }^{25}$

When methylene blue is unavailable, ascorbic acid can be used, although multiple doses are required, and its response is less effective. As an alternative to methylene blue, ascorbic acid can be a therapeutic agent for methemoglobinemia in some cases. ${ }^{19-21,26}$ Ascorbic acid, the most investigated antioxidant and free radical scavenger in the literature, reduces methemoglobin directly. ${ }^{27,28}$ Topal and Topa ${ }^{19}$ reported a case in which methemoglobinemia induced by the local anesthetic prilocaine was treated with ascorbic acid instead of methylene blue as an alternative method. A previously healthy 70-day-old male developed toxic methemoglobinemia after local administration of prilocaine for circumcision, and $300 \mathrm{mg}$ of intravenous ascorbic acid was administered slowly over a 24-hour period. His initial levels of methemoglobin were found to be $24.5 \%$ before ascorbic acid treatment; whereas, at 24 hours after treatment, his methemoglobin levels were $2 \%$. Park et al. ${ }^{20}$ also reported that methemoglobinemia was treated successfully with ascorbic acid. In this case, DDS induced toxic methemoglobinemia, and high doses of ascorbic acid (i.e., $10 \mathrm{~g}$ of ascorbic acid every 6 hours) were administered until approximately 54 hours after emergency department presentation. Another case series of ascorbic acid treatment for methemoglobinemia was reported by Rino et al. ${ }^{26}$ The authors described five cases of children with acquired methemoglobinemia; one patient became symptomatic after treatment with DDS for pemphigus, and three patients developed symptoms after ingestion of either food or water suspected of containing high levels of nitrites. The methemoglobin levels of these patients ranged from $6.4 \%$ to $43 \%$. When intravenous ascorbic acid and 100\% oxygen were provided, the patients recovered successfully. Cimetidine and N-acetylcysteine have also been studied for treating methemoglobinemia; however, their therapeutic effects are poorly understood. ${ }^{8,9,29}$ 
In this study, we demonstrated that intravenous ascorbic acid administration was effective at reducing methemoglobin levels. However, ascorbic acid was slightly less effective than methylene blue administration. At 120 minutes, the methemoglobin concentration of the ascorbic acid group was not significantly different from that of the control group, whereas the methylene blue group showed a more marked difference. In actual clinical cases, because the therapeutic response to ascorbic acid is less marked, repeated doses of intravenous ascorbic acid or an oral preparation have been prescribed, whereas we administered a single intravenous dose of ascorbic acid. ${ }^{20,26}$ Furthermore, we administered $15 \mathrm{mg} / \mathrm{kg}$ of ascorbic acid in doses determined in a pilot study. Therefore, a higher dose of ascorbic acid might be needed to achieve better therapeutic results.

We measured the NO levels and CAT activity in each group to assess the state of oxidative stress. NO is an important regulatory molecule that is associated with host defense and development, and is produced biologically from the oxidation of L-arginine to L-citrulline, which is catalyzed by NO synthase. ${ }^{30} \mathrm{NO}$ is indicative of cytotoxic effects along with other reactive oxygen species, such as $\mathrm{H}_{2} \mathrm{O}_{2}$ and superoxide. ${ }^{31}$ Catalase is an antioxidant enzyme that can degrade $\mathrm{H}_{2} \mathrm{O}_{2}$ to water and oxygen. The breakdown of $\mathrm{H}_{2} \mathrm{O}_{2}$ in the cell by catalase prevents oxidative damage. A number of studies regarding the role of $\mathrm{H}_{2} \mathrm{O}_{2}$ in oxidative stress have been conducted. ${ }^{32,33}$ Contrary to our expectations, plasma NO levels and CAT activity at 60, 120, and 180 minutes did not show any statistical differences among any of the groups. The reasons for this may be 1) the short study period from drug administration until measurement of the drug's effect; 2) the selection of an inappropriate target to measure oxidative stress; and 3) the relatively small dose of administered ascorbic acid (as mentioned earlier). Further study is needed with higher doses of ascorbic acid, as well as with measurements of different oxidative stress parameters. In summary, in the present study, intravenous ascorbic acid administration was effective for the treatment of DDS-induced methemoglobinemia in a murine model.

\section{CONFLICT OF INTEREST}

No potential conflict of interest relevant to this article was reported.

\section{ACKNOWLEDGMENTS}

This research was supported by the grant of Institute of Health Sciences (IHS GNU-2015-02).

\section{REFERENCES}

1. Rodriguez M, Fishman JA. Prevention of infection due to Pneumocystis spp. In human immunodeficiency virus-negative immunocompromised patients. Clin Microbiol Rev 2004;17:77082.

2. Naik PM, Lyon GM 3rd, Ramirez A, et al. Dapsone-induced hemolytic anemia in lung allograft recipients. J Heart Lung Transplant 2008;27:1198-202.

3. Girard PM, Landman R, Gaudebout C, et al. Dapsone-pyrimethamine compared with aerosolized pentamidine as primary prophylaxis against Pneumocystis carinii pneumonia and toxoplasmosis in HIV infection. The PRIO Study Group. N Engl J Med 1993;328:1514-20.

4. Vasconcelles MJ, Bernardo MV, King C, Weller EA, Antin JH. Aerosolized pentamidine as pneumocystis prophylaxis after bone marrow transplantation is inferior to other regimens and is associated with decreased survival and an increased risk of other infections. Biol Blood Marrow Transplant 2000; 6:35-43.

5. Hughes WT. Use of dapsone in the prevention and treatment of Pneumocystis carinii pneumonia: a review. Clin Infect Dis 1998;27:191-204.

6. Thunga G, Sam KG, Patel D, et al. Effectiveness of hemodialysis in acute dapsone overdose: a case report. Am J Emerg Med 2008;26:1070.

7. Mehta M. Cimetidine and dapsone-mediated methaemoglobinaemia. Anaesthesia 2007;62:1188.

8. Coleman MD. Dapsone: modes of action, toxicity and possible strategies for increasing patient tolerance. Br J Dermatol 1993; 129:507-13.

9. Coleman MD, Rhodes $L E$, Scott $A K$, et al. The use of cimetidine to reduce dapsone-dependent methaemoglobinaemia in dermatitis herpetiformis patients. Br J Clin Pharmacol 1992;34: 244-9.

10. Wright RO, Lewander WJ, Woolf AD. Methemoglobinemia: etiology, pharmacology, and clinical management. Ann Emerg Med 1999;34:646-56.

11. Zosel A, Rychter K, Leikin JB. Dapsone-induced methemoglobinemia: case report and literature review. Am J Ther 2007;14: 585-7.

12. Pallais JC, Mackool BT, Pitman MB. Case records of the Massachusetts General Hospital: Case 7-2011: a 52-year-old man with upper respiratory symptoms and low oxygen saturation levels. N Engl J Med 2011;364:957-66.

13. Plotkin JS, Buell JF, Njoku MJ, et al. Methemoglobinemia associated with dapsone treatment in solid organ transplant re- 
cipients: a two-case report and review. Liver Transpl Surg 1997; 3:149-52

14. Turner MD, Karlis V, Glickman RS. The recognition, physiology, and treatment of medication-induced methemoglobinemia: a case report. Anesth Prog 2007;54:115-7.

15. Park KH, Kim H, Lee CC, et al. Dapsone intoxication: clinical course and characteristics. Clin Toxicol (Phila) 2010;48:51621.

16. Kang $\mathrm{KS}$, Kim $\mathrm{HI}$, Kim $\mathrm{OH}$, et al. Clinical outcomes of adverse cardiovascular events in patients with acute dapsone poisoning. Clin Exp Emerg Med 2016;3:41-5.

17. Youngster I, Arcavi L, Schechmaster $R$, et al. Medications and glucose-6-phosphate dehydrogenase deficiency: an evidencebased review. Drug Saf 2010;33:713-26.

18. Sills MR, Zinkham WH. Methylene blue-induced Heinz body hemolytic anemia. Arch Pediatr Adolesc Med 1994;148:30610.

19. Topal H, Topal Y. Toxic methemoglobinemia treated with ascorbic Acid: case report. Iran Red Crescent Med J 2013;15:e12718.

20. Park SY, Lee KW, Kang TS. High-dose vitamin C management in dapsone-induced methemoglobinemia. Am J Emerg Med 2014;32:684.

21. D'sa SR, Victor P, Jagannati M, Sudarsan TI, Carey RA, Peter JV. Severe methemoglobinemia due to ingestion of toxicants. Clin Toxicol (Phila) 2014;52:897-900.

22. Jo YH, Kwon WY, Lee JH, et al. The effect of ethyl pyruvate on dapsone-induced methemoglobinemia in rats. Clin Toxicol (Phila) 2008;46:811-4.

23. Kane GC, Hoehn SM, Behrenbeck TR, Mulvagh SL. Benzocaineinduced methemoglobinemia based on the Mayo Clinic experience from 28478 transesophageal echocardiograms: incidence, outcomes, and predisposing factors. Arch Intern Med 2007;167:1977-82.

24. Shopes E, Gerard W, Baughman J. Methylene blue encephalopathy: a case report and review of published cases. AANA J
2013;81:215-21.

25. Top WM, Gillman PK, de Langen CJ, Kooy A. Fatal methylene blue associated serotonin toxicity. Neth J Med 2014;72:17981.

26. Rino PB, Scolnik D, Fustinana $A$, Mitelpunkt $A$, Glatstein $M$. Ascorbic acid for the treatment of methemoglobinemia: the experience of a large tertiary care pediatric hospital. Am J Ther 2014;21:240-3.

27. Wilson JX. Regulation of vitamin C transport. Annu Rev Nutr 2005;25:105-25.

28. Dotsch J, Demirakca S, Cryer A, Hanze J, Kuhl PG, Rascher W. Reduction of NO-induced methemoglobinemia requires extremely high doses of ascorbic acid in vitro. Intensive Care Med 1998;24:612-5.

29. Tanen DA, LoVecchio F, Curry SC. Failure of intravenous N-acetylcysteine to reduce methemoglobin produced by sodium nitrite in human volunteers: a randomized controlled trial. Ann Emerg Med 2000;35:369-73.

30. Hobbs AJ, Fukuto JM, Ignarro $\sqcup$. Formation of free nitric oxide from I-arginine by nitric oxide synthase: direct enhancement of generation by superoxide dismutase. Proc Natl Acad Sci U S A 1994;91:10992-6.

31. Rubbo $H$, Radi $R$, Trujillo $M$, et al. Nitric oxide regulation of superoxide and peroxynitrite-dependent lipid peroxidation. Formation of novel nitrogen-containing oxidized lipid derivatives. J Biol Chem 1994;269:26066-75.

32. Bai J, Rodriguez AM, Melendez JA, Cederbaum Al. Overexpression of catalase in cytosolic or mitochondrial compartment protects HepG2 cells against oxidative injury. J Biol Chem 1999 274:26217-24.

33. Tome ME, Baker AF, Powis G, Payne CM, Briehl MM. Catalaseoverexpressing thymocytes are resistant to glucocorticoid-induced apoptosis and exhibit increased net tumor growth. Cancer Res 2001;61:2766-73. 